\title{
The exponent of the homotopy groups of Moore spectra and the stable Hurewicz homomorphism
}

\author{
Dominique Arlettaz
}

Short title: The stable Hurewicz homomorphism

\begin{abstract}
This paper shows that for the Moore spectrum $M G$ associated with any abelian group $G$, and for any positive integer $n$, the order of the Postnikov $k$-invariant $k^{n+1}(M G)$ is equal to the exponent of the homotopy group $\pi_{n} M G$. In the case of the sphere spectrum $S$, this implies that the exponents of the homotopy groups of $S$ provide a universal estimate for the exponent of the kernel of the stable Hurewicz homomorphism $h_{n}: \pi_{n} X \rightarrow$ $E_{n}(X)$ for the homology theory $E_{*}(-)$ corresponding to any connective ring spectrum $E$ such that $\pi_{0} E$ is torsion-free and for any bounded below spectrum $X$. Moreover, an upper bound for the exponent of the cokernel of the generalized Hurewicz homomorphism $h_{n}: E_{n}(X) \rightarrow H_{n}\left(X ; \pi_{0} E\right)$, induced by the 0 -th Postnikov section of $E$, is obtained for any connective spectrum $E$. An application of these results enables us to approximate in a universal way both kernel and cokernel of the unstable Hurewicz homomorphism between the algebraic K-theory of any ring and the ordinary integral homology of its linear group.
\end{abstract}

1991 Mathematics subject classification: Primary 55 N 20, 55 P 42.

Secondary 19 D 55, 55 Q 45, 55 S 45. 


\section{Introduction}

The first purpose of this paper is to show that for the Moore spectrum $M G$ associated with any abelian group $G$, and for any positive integer $n$, the order of the Postnikov $k$ invariant $k^{n+1}(M G)$ is exactly equal to the exponent of the homotopy group $\pi_{n} M G$ (see Theorem 1.3).

This equality, in the case of the sphere spectrum $S=M \mathbb{Z}$, enables us to prove that the exponents of the homotopy groups of $S$ provide a universal approximation of the exponent of the kernel of the Hurewicz homomorphism

$$
h_{n}: \pi_{n} X \longrightarrow E_{n}(X)
$$

for the homology theory $E_{*}(-)$ corresponding to any connective (i.e., (-1)-connected) ring spectrum $E$ (for which we assume that $\pi_{0} E$ is torsion-free) and for any bounded below spectrum $X$. More precisely, we obtain the following result (see Theorem 2.2):

For any $(b-1)$-connected spectrum $X$ and any integer $n \geq b+1$, if $x$ is an element of the kernel of $h_{n}: \pi_{n} X \rightarrow E_{n}(X)$, then $\left(\rho_{1} \rho_{2} \cdots \rho_{n-b}\right) x \otimes 1=0$ in $\pi_{n} X \otimes \pi_{0} E$, where $\rho_{j}$ denotes the exponent of $\pi_{j} S$ for $j \geq 1$.

It is also possible to define a generalized Hurewicz homomorphism

$$
h_{n}: E_{n}(X) \longrightarrow H_{n}\left(X ; \pi_{0} E\right)
$$

for any connective spectrum $E$ and for any spectrum $X$. The next goal of the paper is to give a universal upper bound for the exponent of the cokernel of this homomorphism (see Theorem 3.2):

For any $(b-1)$-connected spectrum $X$ and any integer $n \geq b+2$, the cokernel of $h_{n}: E_{n}(X) \rightarrow H_{n}\left(X ; \pi_{0} E\right)$ satisfies $\left(\rho_{1}(E) \rho_{2}(E) \cdots \rho_{n-b-1}(E)\right)\left(\right.$ coker $\left.h_{n}\right)=0$, where $\rho_{j}(E)$ is the order of the $k$-invariant $k^{j+1}(E)$ for $j \geq 1$.

In the case of the classical stable Hurewicz homomorphism

$$
h_{n}: \pi_{n} X \longrightarrow H_{n}(X ; \mathbb{Z})
$$

for a $(b-1)$-connected spectrum $X$, it is known that $h_{n}$ is an isomorphism if $n=b$ and an epimorphism if $n=b+1$. The above general results imply that the exponents 
$\rho_{j}$ of the homotopy groups $\pi_{j} S$ of the sphere spectrum $S(j \geq 1)$ produce universal exponents for both kernel and cokernel of $h_{n}$ (see Theorem 4.1):

For any $(b-1)$-connected spectrum $X$, the classical stable Hurewicz homomorphism $h_{n}: \pi_{n} X \rightarrow H_{n}(X ; \mathbb{Z})$ has the following properties:

(a) $\left(\rho_{1} \rho_{2} \cdots \rho_{n-b}\right)\left(\operatorname{ker} h_{n}\right)=0$ for all integers $n \geq b+1$,

(b) $\left(\rho_{1} \rho_{2} \cdots \rho_{n-b-1}\right)\left(\right.$ coker $\left.h_{n}\right)=0$ for all integers $n \geq b+2$.

This important fact does not seem to be well-known, although its proof is quite simple. Notice that these theorems hold without any finiteness condition on the spectrum $X$.

It is for instance interesting to apply these results to the $n$-dimensional classical unstable Hurewicz homomorphism between the algebraic K-theory of a ring $R$ and the ordinary integral homology of the group $E(R)$ generated by elementary matrices with coefficients in $R$ (see Corollaries 5.1 and 5.2): for $n \geq 3$ its kernel is annihilated by the integer $\rho_{1} \rho_{2} \cdots \rho_{n-2}$, which is independent of $R$ (this extends the results of [2]), and we get a relationship between its cokernel and the iterated homology suspension $\sigma_{n}: H_{n}(E(R) ; \mathbb{Z}) \rightarrow H_{n}\left(X_{B E(R)^{+}} ; \mathbb{Z}\right)$, where $X_{B E(R)^{+}}$is a 1-connected $\Omega$-spectrum associated with the infinite loop space $B E(R)^{+}$.

The paper is organized as follows. In Section 1, we establish the correspondence between the exponent of the homotopy groups of a Moore spectrum and the order of its $k$-invariants. The second section is devoted to the study of the kernel of the Hurewicz homomorphism $h_{*}: \pi_{*} X \rightarrow E_{*}(X)$ for a connective ring spectrum $E$. In Section 3, we define the generalized Hurewicz homomorphism $h_{*}: E_{*}(X) \rightarrow H_{*}\left(X ; \pi_{0} E\right)$, for a connective spectrum $E$, and investigate its cokernel. Section 4 formulates the previous results in the case of the classical stable Hurewicz homomorphism and presents their consequences on the classical unstable Hurewicz homomorphism for infinite loop spaces. The relation between algebraic K-theory and linear group homology is then discussed in Section 5. Finally, we mention in Section 6 the anologous assertions for the Hurewicz homomorphism with finite coefficients.

Throughout the paper, we are working in the category of CW-spectra, and we denote by $H(G)$ the Eilenberg-MacLane spectrum having all homotopy groups trivial except for $G$ in dimension 0 and, for a spectrum $E$ and an integer $m$, by $\alpha_{m}: E \rightarrow E[m]$ its $m$-th Postnikov section (i.e., $\pi_{j} E[m]=0$ for $j>m$ and $\left(\alpha_{m}\right)_{*}: \pi_{j} E \stackrel{\cong}{\longrightarrow} \pi_{j} E[m]$ for $j \leq m)$.

I would like to thank Paul Goerss for useful comments. 


\section{The Postnikov invariants of Moore spectra}

Let $G$ be an abelian group and $M G$ the corresponding Moore spectrum (see [1], p. 200): $M G$ is a connective spectrum such that $H_{0}(M G ; \mathbb{Z}) \cong G$ and $H_{j}(M G ; \mathbb{Z})=0$ for $j \geq 1$. The Postnikov $k$-invariants of the spectrum $M G$ are cohomology classes $k^{n+1}(M G) \in H^{n+1}\left(M G[n-1] ; \pi_{n} M G\right)$, for $n \geq 1$. Since $M G$ is connective, all its $k$-invariants are torsion classes according to Theorem 1.5 of [3].

Proposition 1.1. If $X$ is a $(b-1)$-connected spectrum and $n$ an integer $\geq b+1$, then the classical Hurewicz homomorphism $h_{n}: \pi_{n} X \rightarrow H_{n}(X ; \mathbb{Z})$ satisfies $\rho_{n}(X)\left(\operatorname{ker} h_{n}\right)=0$, where $\rho_{n}(X)$ is the order of the $k$-invariant $k^{n+1}(X)$ in $H^{n+1}\left(X[n-1] ; \pi_{n} X\right)$.

Proof. Since $X$ is bounded below, $\rho_{n}(X)$ is finite and there exists a map of spectra $f_{n}: X \rightarrow \Sigma^{n} H\left(\pi_{n} X\right)$ inducing multiplication by $\rho_{n}(X)$ on $\pi_{n} X$ (see [3], Lemma 1.1 and Theorem 1.5). This map and the Hurewicz homomorphism produce the commutative diagram

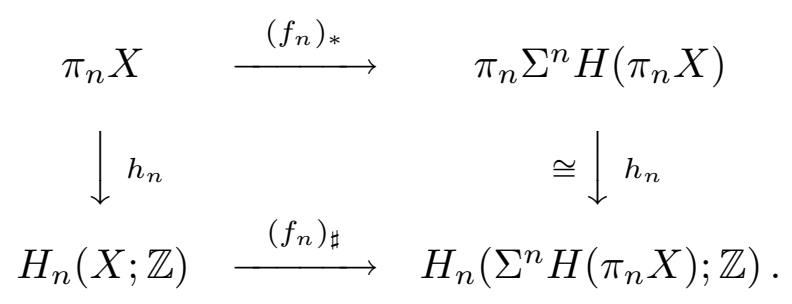

Therefore, the kernel of $h_{n}: \pi_{n} X \rightarrow H_{n}(X ; \mathbb{Z})$ is a subgroup of the kernel of the homomorphism $\left(f_{n}\right)_{*}$ which is multiplication by $\rho_{n}(X)$, and consequently, $\rho_{n}(X)\left(\right.$ ker $\left.h_{n}\right)=0$.

Definition 1.2. For $n \geq 1$, let us call $\rho_{n}(G)$ the order of the $k$-invariant $k^{n+1}(M G)$ in the cohomology group $H^{n+1}\left(M G[n-1] ; \pi_{n} M G\right)$.

We may deduce from Proposition 1.1 the main result of this section.

Theorem 1.3. For any abelian group $G$ and any positive integer $n, \rho_{n}(G)$ is equal to the exponent of the homotopy group $\pi_{n} M G$.

Proof. Since $n \geq 1$, the group $H_{n}(M G ; \mathbb{Z})$ vanishes and Proposition 1.1 implies that $\rho_{n}(G) \pi_{n} M G=0$. On the other hand, it is clear that $\rho_{n}(G)$ divides the exponent of $\pi_{n} M G$, because $k^{n+1}(M G)$ belongs to the group $H^{n+1}\left(M G[n-1] ; \pi_{n} M G\right)$. 
Example 1.4. For the sphere spectrum $S=M \mathbb{Z}$, the order $\rho_{n}(\mathbb{Z})$ of the $k$-invariant $k^{n+1}(S)$ is exactly the exponent of the group $\pi_{n} S=\lim _{k} \pi_{n+k} S^{k}$, for all $n \geq 1$. In the remainder of the paper, we shall write $\rho_{n}$ for $\rho_{n}(\mathbb{Z})$.

Remark 1.5. For any abelian group $G$, since $M G$ is the cofibre of a map from a wedge of sphere spectra to a wedge of sphere spectra (see [1], p. 200), the homotopy exact sequence of this cofibration shows that the exponent of $\pi_{n} M G$ is bounded by the product $\rho_{n} \rho_{n-1}$, in other words that $\rho_{n}(G)$ divides $\rho_{n} \rho_{n-1}$ for any abelian group $G$ and for all integers $n \geq 2$ (it is clear that $\rho_{1}(G)=1$ or 2 ).

Remark 1.6. The assertion of Theorem 1.3 holds also for any Moore space (instead of spectrum), having only one non-trivial reduced integral homology group in dimension $\ell$, assuming we are looking at an integer $n \geq \ell+1$ such that the $n$-th homotopy group of the Moore space has finite exponent. However, observe that if this exponent is infinite, then the order of the corresponding $k$-invariant of the Moore space is also infinite.

It is possible to generalize the statement of Theorem 1.3 as follows.

Proposition 1.7. Let $X$ be a connective spectrum with the property that $H_{j}(X ; \mathbb{Z})$ is a group of finite exponent $\eta_{j}$ for all $j \geq 1$. Then, for any positive integer $n$, the exponent of $\pi_{n} X$ is a positive multiple of the order $\rho_{n}(X)$ of the $k$-invariant $k^{n+1}(X)$ and divides the product $\rho_{n}(X) \eta_{n}$.

Proof. If $x$ is an element of $\pi_{n} X$, then $\eta_{n} x$ lies in the kernel of $h_{n}: \pi_{n} X \rightarrow H_{n}(X ; \mathbb{Z})$ and one obtains from Proposition 1.1 the equality $\left(\rho_{n}(X) \eta_{n}\right) x=0$. As mentioned in the proof of Theorem 1.3, it is again trivial that the exponent of $\pi_{n} X$ is a positive multiple of $\rho_{n}(X)$.

\section{The kernel of the Hurewicz homomorphism}

Let $E$ be a connective ring spectrum with identity $i: S \rightarrow E$. For a spectrum $X$ and an integer $n$, the E-Hurewicz homomorphism is

$$
h_{n}: \pi_{n} X=\pi_{n}(S \wedge X) \stackrel{(i \wedge \mathrm{id})_{*}}{\longrightarrow} \pi_{n}(E \wedge X)=E_{n}(X),
$$


where $E_{*}(-)$ is the homology theory associated with the spectrum $E$ and id $X \rightarrow X$ the identity (see [1], p. 58 or [9], p. 290). In the case of the spectrum $E=H(\mathbb{Z}), h_{n}$ is the classical Hurewicz homomorphism

$$
h_{n}: \pi_{n} X \longrightarrow H_{n}(X ; \mathbb{Z})
$$

The objective of this section is to use the result on the sphere spectrum described in Example 1.4, in order to approximate the exponent of the kernel of the E-Hurewicz homomorphism $h_{n}$ in a universal way. Notice that, in general, the cokernel of $h_{n}$ may be of infinite exponent. For technical reason (see the proof of Theorem 2.2), we shall concentrate our attention to spectra $E$ for which $\pi_{0} E$ is torsion-free.

Definition 2.1. For $m \geq 1$, let $\bar{\rho}_{m}=\prod_{j=1}^{m} \rho_{j}$, where $\rho_{j}=\rho_{j}(\mathbb{Z})$ is the exponent of $\pi_{j} S$. Notice that a prime number $p$ divides $\bar{\rho}_{m}$ if and only if $p \leq \frac{m+3}{2}$.

Theorem 2.2. Let $E$ be a connective ring spectrum such that $\pi_{0} E$ is torsion-free and $X$ any $(b-1)$-connected spectrum. The kernel of the E-Hurewicz homomorphism $h_{n}$ : $\pi_{n} X \rightarrow E_{n}(X)$ has the following property: if $x$ is an element of ker $h_{n}$, then

$$
\bar{\rho}_{n-b} x \otimes 1=0 \text { in } \pi_{n} X \otimes \pi_{0} E
$$

for all integers $n \geq b+1$.

Proof. By Lemma 4.1 of [3], $\pi_{n} X=\pi_{n}(S \wedge X) \cong S[n-b]_{n}(S \wedge X)$ and similarly $E_{n}(X)=\pi_{n}(E \wedge X) \cong S[n-b]_{n}(E \wedge X)$, since the spectra $S \wedge X$ and $E \wedge X$ are $(b-1)$ connected. Because of Theorem 1.3, the method developed in [4] shows the existence of maps of spectra

$$
S[n-b] \stackrel{\varphi}{\longrightarrow} \bigvee_{t=0}^{n-b} \Sigma^{t} H\left(\pi_{t} S\right) \stackrel{\psi}{\longrightarrow} S[n-b]
$$

such that the composition $\psi \varphi$ is homotopic to the $\left(\bar{\rho}_{n-b}\right)$-th power map: $S[n-b] \rightarrow$ $S[n-b]$; for any bounded below spectrum $Y$, they induce homomorphisms

$$
S[n-b]_{n}(Y) \stackrel{\Phi}{\longrightarrow}\left(\bigvee_{t=0}^{n-b} \Sigma^{t} H\left(\pi_{t} S\right)\right)_{n}(Y) \cong \bigoplus_{t=0}^{n-b} H_{n-t}\left(Y ; \pi_{t} S\right) \stackrel{\Psi}{\longrightarrow} S[n-b]_{n}(Y)
$$

such that the composition $\Psi \Phi$ is multiplication by $\bar{\rho}_{n-b}$ (see Corollary 1.4 and Theorem 2.2 of [4]). Now, consider the commutative diagram 


$$
\begin{array}{ccc}
\pi_{n} X \cong S[n-b]_{n}(S \wedge X) & \stackrel{h_{n}=(i \wedge \mathrm{id})_{*}}{\longrightarrow} & S[n-b]_{n}(E \wedge X) \cong E_{n}(X) \\
& \stackrel{(i \wedge \mathrm{id})_{*}}{\longrightarrow} & \bigoplus_{t=0}^{n-b} H_{n-t}\left(E \wedge X ; \pi_{t} S\right) .
\end{array}
$$

For any $t$ with $0 \leq t \leq n-b$, the homomorphism $(i \wedge \text { id })_{*}: H_{n-t}(S \wedge X ; \mathbb{Z}) \rightarrow$ $H_{n-t}(E \wedge X ; \mathbb{Z})$ is the composition of $i_{\sharp}: H_{n-t}(X ; \mathbb{Z}) \rightarrow H_{n-t}(X ; \mathbb{Z}) \otimes \pi_{0} E$ (induced by $\left.i_{*}: \pi_{0} S \cong \mathbb{Z} \rightarrow \pi_{0} E\right)$ with a split injection $H_{n-t}(X ; \mathbb{Z}) \otimes \pi_{0} E \hookrightarrow H_{n-t}(E \wedge X ; \mathbb{Z})$; since $\pi_{0} E$ is torsion-free, the same is true for the bottom horizontal arrow in the above diagram. Therefore, if $x \in \operatorname{ker} h_{n}$, then $\Phi(x)$ vanishes under the top horizontal map in the next commutative diagram:

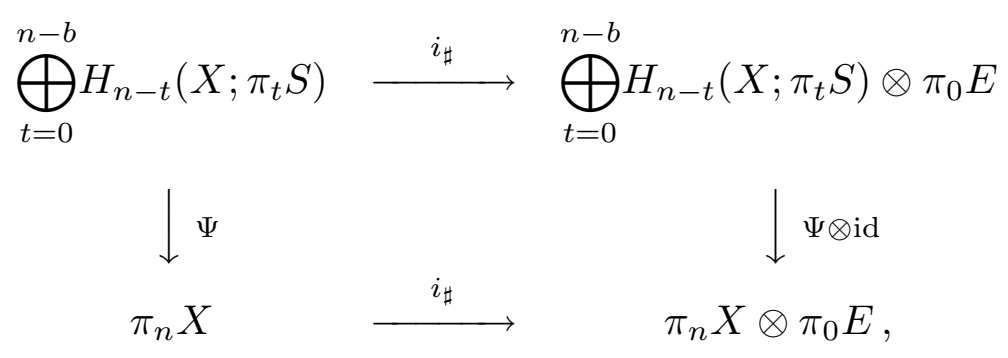

and we conclude that $\bar{\rho}_{n-b} x=\Psi \Phi(x)$ belongs to the kernel of the bottom horizontal homomorphism $i_{\sharp}$.

Corollary 2.3. If $E$ is a connective ring spectrum with $\pi_{0} E \cong \mathbb{Z}$ and $X$ any $(b-1)$ connected spectrum, then the kernel of the E-Hurewicz homomorphism $h_{n}: \pi_{n} X \rightarrow$ $E_{n}(X)$ satisfies

$$
\bar{\rho}_{n-b}\left(\operatorname{ker} h_{n}\right)=0
$$

for all integers $n \geq b+1$.

Remark 2.4. In the classical case (where $E=H(\mathbb{Z})$ ), another way to prove this corollary may be deduced from the Atiyah-Hirzebruch spectral sequence argument which will be presented in Lemma 3.3 and Remark 3.4. 
Remark 2.5. It is sometimes possible to get a better bound on the exponent of the kernel of $h_{n}$. Suppose that $\pi_{0} E \cong \mathbb{Z}$ and let $X$ be a $(b-1)$-connected spectrum, $n$ an integer $\geq b+1$ and $\rho_{n}(X)$ the order of the $k$-invariant $k^{n+1}(X)$ in $H^{n+1}\left(X[n-1] ; \pi_{n} X\right)$, then the argument of the proof of Proposition 1.1 shows that the $E$-Hurewicz homomorphism $h_{n}: \pi_{n} X \rightarrow E_{n}(X)$ fulfills

$$
\rho_{n}(X)\left(\operatorname{ker} h_{n}\right)=0 \text {. }
$$

Consequently, we may actually improve the statement of Corollary 2.3 as follows:

$$
\operatorname{gcd}\left(\bar{\rho}_{n-b}, \rho_{n}(X)\right)\left(\operatorname{ker} h_{n}\right)=0 .
$$

Recall that we have defined in [2] and [3] integers $R_{j}$, for $j \geq 2$, with the property that $\rho_{n}(X)$ divides $R_{n-b+1}$ for any $(b-1)$-connected spectrum $X$. Thus, we can even replace $\operatorname{gcd}\left(\bar{\rho}_{n-b}, \rho_{n}(X)\right)$ by $\operatorname{gcd}\left(\bar{\rho}_{n-b}, R_{n-b+1}\right)$.

Theorem 2.2 has a direct consequence on the Hurewicz homomorphism for spaces. If $K$ is a CW-complex, let us consider its suspension spectrum $\Sigma^{\infty}(K)$ and the iterated suspension $s_{n}: \pi_{n} K \rightarrow \pi_{n} \Sigma^{\infty}(K)$.

Corollary 2.6. Let $E$ be a connective ring spectrum such that $\pi_{0} E$ is torsion-free, $\widetilde{E}_{*}(-)$ the corresponding reduced homology theory, and $K$ a $(b-1)$-connected $C W$ complex with $b \geq 1$. For any integer $n \geq b+1$, if $x \in \operatorname{ker} h_{n}: \pi_{n} K \rightarrow \widetilde{E}_{n}(K)$, then $s_{n}\left(\bar{\rho}_{n-b} x\right) \otimes 1=0$ in $\pi_{n} \Sigma^{\infty}(K) \otimes \pi_{0} E$.

Proof. The commutativity of the diagram

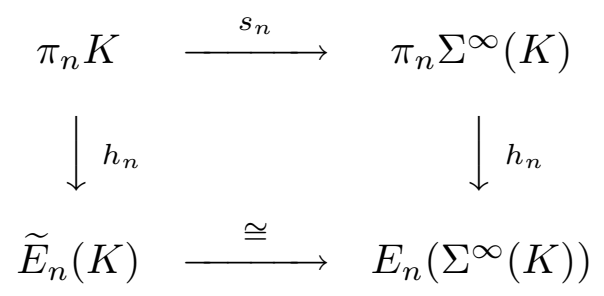

shows that if $x$ is an element of the kernel of $h_{n}: \pi_{n} K \rightarrow \widetilde{E}_{n}(K)$, then $s_{n}(x)$ belongs to the kernel of $h_{n}: \pi_{n} \Sigma^{\infty}(K) \rightarrow E_{n}\left(\Sigma^{\infty}(K)\right)$ and $s_{n}\left(\bar{\rho}_{n-b} x\right) \otimes 1=\bar{\rho}_{n-b} s_{n}(x) \otimes 1=0$ in $\pi_{n} \Sigma^{\infty}(K) \otimes \pi_{0} E$ by Theorem 2.2 .

If $n \leq 2(b-1)$, then $s_{n}$ is an isomorphism by the Freudenthal suspension theorem and one obtains the following statement. 
Corollary 2.7. Let $E$ be a connective ring spectrum such that $\pi_{0} E$ is torsion-free, $\widetilde{E}_{*}(-)$ the corresponding reduced homology theory, $K$ a $(b-1)$-connected $C W$-complex with $b \geq 3$, and $n$ an integer such that $b+1 \leq n \leq 2(b-1)$. If $x \in \operatorname{ker} h_{n}: \pi_{n} K \rightarrow$ $\widetilde{E}_{n}(K)$, then $\bar{\rho}_{n-b} x \otimes 1=0$ in $\pi_{n} K \otimes \pi_{0} E$.

Let us conclude this section by recalling that it is actually possible to define a more general E-Hurewicz homomorphism $h_{n}: F_{n}(X) \rightarrow(E \wedge F)_{n}(X)$, for a connective ring spectrum $E$ and spectra $F$ and $X$, and for all integers $n$, as follows (see [9], p. 290):

$$
h_{n}: F_{n}(X)=\pi_{n}(F \wedge X)=\pi_{n}(S \wedge F \wedge X) \stackrel{(i \wedge \mathrm{id} \wedge \mathrm{id})_{*}}{\longrightarrow} \pi_{n}(E \wedge F \wedge X)=(E \wedge F)_{n}(X) .
$$

We may extend the above results to this homomorphism.

Corollary 2.8. Let $E$ be a connective ring spectrum such that $\pi_{0} E$ is torsion-free, $F$ a $(c-1)$-connected spectrum and $X$ a $(b-1)$-connected spectrum. The E-Hurewicz homomorphism $h_{n}: F_{n}(X) \rightarrow(E \wedge F)_{n}(X)$ has the following property: if $x$ is an element of ker $h_{n}$, then $\bar{\rho}_{n-b-c} x \otimes 1=0$ in $F_{n}(X) \otimes \pi_{0} E$ for all integers $n \geq b+c+1$.

Proof. The homomorphism $h_{n}$ is in fact the Hurewicz homorphism $h_{n}: \pi_{n}(F \wedge X) \rightarrow$ $E_{n}(F \wedge X)$. Thus, the assertion is just the application of Theorem 2.2 to the $(b+c-1)$ connected spectrum $F \wedge X$.

\section{The cokernel of the generalized Hurewicz homomorphism}

Now, consider a connective spectrum $E$ and its associated homology theory $E_{*}(-)$. For a spectrum $X$ and an integer $n$, we can define a generalized E-Hurewicz homomorphism

$$
h_{n}: E_{n}(X) \longrightarrow H_{n}\left(X ; \pi_{0} E\right)
$$

as follows:

$$
h_{n}: E_{n}(X)=\pi_{n}(E \wedge X) \stackrel{\left(\alpha_{0} \wedge \mathrm{id}\right)_{*}}{\longrightarrow} \pi_{n}\left(H\left(\pi_{0} E\right) \wedge X\right)=H_{n}\left(X ; \pi_{0} E\right),
$$

where $\alpha_{0}: E \rightarrow E[0]=H\left(\pi_{0} E\right)$ denotes the 0-th Postnikov section of the spectrum $E$ and id $: X \rightarrow X$ the identity. In the case where $E$ is the sphere spectrum $S$, $\alpha_{0}: S \rightarrow H(\mathbb{Z})$ is a generator of $\pi_{0} H(\mathbb{Z})$ and $h_{n}$ is the classical Hurewicz homomorphism

$$
h_{n}: \pi_{n} X \longrightarrow H_{n}(X ; \mathbb{Z})
$$


If $X$ is $(b-1)$-connected, it is obvious that the generalized $E$-Hurewicz homomorphism $h_{n}: E_{n}(X) \rightarrow H_{n}\left(X ; \pi_{0} E\right)$ is an isomorphism if $n=b$ and an epimorphism if $n=b+1$. In general, the kernel of $h_{n}$ may be of infinite exponent when $n \geq b+1$. The purpose of this section is to show that the study of the order of the $k$-invariants of $E$ provides universal upper bounds for the exponent of its cokernel in all dimensions $n$.

Definition 3.1. For $m \geq 1$, let $\bar{\rho}_{m}(E)=\prod_{j=1}^{m} \rho_{j}(E)$, where $\rho_{j}(E)$ is the order of the $k$-invariant $k^{j+1}(E)$ in $H^{j+1}\left(E[j-1] ; \pi_{j} E\right)$. Remember that $\rho_{j}(E)$ is always finite according to [3], Theorem 1.5. If $E=S, \bar{\rho}_{m}(E)=\bar{\rho}_{m}$ because of Theorem 1.3.

Let $X$ be a $(b-1)$-connected spectrum and $n$ an integer $\geq b+2$. Notice first that $E_{n}(X) \cong E[n-b]_{n}(X)$ by Lemma 4.1 of [3]: thus, we may replace $E$ by $E[n-b]$ and consider $\alpha_{0}: E[n-b] \rightarrow H\left(\pi_{0} E\right)$. Again, it is possible to use the method introduced in [4] to construct a map of spectra $\theta_{n}: H\left(\pi_{0} E\right) \rightarrow E[n-b]$ such that the composition

$$
H\left(\pi_{0} E\right) \stackrel{\theta_{n}}{\longrightarrow} E[n-b] \stackrel{\alpha_{0}}{\longrightarrow} H\left(\pi_{0} E\right)
$$

is homotopic to the $\left(\bar{\rho}_{n-b}(E)\right)$-th power map: $H\left(\pi_{0} E\right) \rightarrow H\left(\pi_{0} E\right)$; consequently, we obtain a homomorphism $\Theta_{n}: H_{n}\left(X ; \pi_{0} E\right) \rightarrow E[n-b]_{n}(X) \cong E_{n}(X)$ with the property that the composition

$$
H_{n}\left(X ; \pi_{0} E\right) \stackrel{\Theta_{n}}{\longrightarrow} E_{n}(X) \stackrel{h_{n}}{\longrightarrow} H_{n}\left(X ; \pi_{0} E\right)
$$

is multiplication by $\bar{\rho}_{n-b}(E)$ on $H_{n}\left(X ; \pi_{0} E\right)$ (see Theorems 1.5 and 2.4 of [4]). Therefore, we may conclude that

$$
\bar{\rho}_{n-b}(E)\left(\operatorname{coker} h_{n}\right)=0 .
$$

But, it turns out that we can get a slightly better estimate of the exponent of the cokernel of $h_{n}$.

Theorem 3.2. For any connective spectrum $E$ and any $(b-1)$-connected spectrum $X$, the cokernel of the generalized E-Hurewicz homomorphism $h_{n}: E_{n}(X) \rightarrow H_{n}\left(X ; \pi_{0} E\right)$ satisfies

$$
\bar{\rho}_{n-b-1}(E)\left(\operatorname{coker} h_{n}\right)=0
$$

for all integers $n \geq b+2$.

The proof of this theorem is based on the following 
Lemma 3.3. In the Atiyah-Hirzebruch spectral sequence $E_{s, t}^{2} \cong H_{s}\left(X ; \pi_{t} E\right) \Rightarrow E_{s+t}(X)$ converging towards the E-homology of any $(b-1)$-connected spectrum $X$, the edge homomorphism $E_{n}(X) \rightarrow E_{n, 0}^{\infty} \hookrightarrow E_{n, 0}^{2} \cong H_{n}\left(X ; \pi_{0} E\right)$ is exactly the generalized $E$ Hurewicz homomorphism $h_{n}$, for all integers $n \geq b$.

Proof of Lemma 3.3. For an integer $\ell$ with $0 \leq \ell \leq n-b$, let us denote the fibre of the Postnikov section $\alpha_{\ell-1}: E[n-b] \rightarrow E[\ell-1]$ by $E(\ell, n-b]$ and the inclusion $E(\ell, n-b] \hookrightarrow$ $E[n-b]$ by $\beta_{\ell}(E(\ell, n-b]$ is the spectrum obtained from $E[n-b]$ by killing its homotopy groups in dimensions $<\ell$ ). The convergence of the Atiyah-Hirzebruch spectral sequence implies the existence of a filtration $0 \subset F_{0} \subset F_{1} \subset \cdots \subset F_{n-b}=E_{n}(X) \cong E[n-b]_{n}(X)$, such that $F_{j} / F_{j-1} \cong E_{j+b, n-b-j}^{\infty}$, which is given by (see Section 4 of [10]):

$$
F_{j}=\operatorname{image}\left(E(n-b-j, n-b]_{n}(X) \stackrel{\left(\beta_{n-b-j}\right)_{*}}{\longrightarrow} E[n-b]_{n}(X)\right) \text { for } 0 \leq j \leq n-b .
$$

In particular, $F_{n-b-1}=$ image $\left(\left(\beta_{1}\right)_{*}: E(1, n-b]_{n}(X) \rightarrow E[n-b]_{n}(X)\right)$ is isomorphic to the kernel of the homomorphism $h_{n}: E_{n}(X) \cong E[n-b]_{n}(X) \rightarrow E[0]_{n}(X) \cong H_{n}\left(X ; \pi_{0} E\right)$ induced by $\alpha_{0} \wedge$ id, because

$$
E(1, n-b] \stackrel{\beta_{1}}{\longrightarrow} E[n-b] \stackrel{\alpha_{0}}{\longrightarrow} E[0]
$$

is a cofibration. It then clearly follows that $E_{n, 0}^{\infty} \cong$ image $h_{n}$.

Remark 3.4. This lemma also shows that if the homotopy groups $\pi_{j}(E)$ have finite exponents $e_{j}$ for $j \geq 1$, then the product $e_{1} e_{2} \cdots e_{n-b}$ is an upper bound for the exponent of the kernel of $h_{n}: E_{n}(X) \rightarrow H_{n}\left(X ; \pi_{0} E\right)$. In the case of the classical Hurewicz homomorphism $h_{n}: \pi_{n} X \rightarrow H_{n}(X ; \mathbb{Z})$, this gives a simple proof of Corollary 2.3.

Proof of Theorem 3.2. Since $X$ is $(b-1)$-connected, the Atiyah-Hirzebruch spectral sequence $E_{s, t}^{2} \cong H_{s}\left(X ; \pi_{t} E\right) \Rightarrow E_{s+t}(X)$ has the property that $E_{s, t}^{*}=0$ if $s<b$ and $E_{n, 0}^{\infty}=E_{n, 0}^{n-b+1}$. According to the previous lemma, the image of $h_{n}$ is $E_{n, 0}^{n-b+1}$, which is the subgroup of $E_{n, 0}^{2} \cong H_{n}\left(X ; \pi_{0} E\right)$ consisting of the kernel of the differentials $d_{n, 0}^{r}: E_{n, 0}^{r} \rightarrow E_{n-r, r-1}^{r}$ for $2 \leq r \leq n-b$, and coker $h_{n} \cong E_{n, 0}^{2} / E_{n, 0}^{n-b+1}$. However, it follows from Proposition 2.1 of [3] that $\rho_{r-1}(E) d_{n, 0}^{r}=0$. This implies inductively that the exponent of coker $h_{n}$ is bounded by the product $\rho_{1}(E) \rho_{2}(E) \cdots \rho_{n-b-1}(E)=\bar{\rho}_{n-b-1}(E)$.

Example 3.5. Consider the Brown-Peterson spectrum $B P$ associated with a prime number $p$ and the generalized $B P$-Hurewicz homomorphism $h_{n}: B P_{n}(X) \longrightarrow H_{n}\left(X ; \mathbb{Z}_{(p)}\right)$. It is easy to check that the kernel of $h_{n}$ may be of infinite exponent and, on the other hand, to deduce the following result on the cokernel of $h_{n}$ from Theorem 3.2 together with the determination of the order of the $k$-invariants of $B P$ presented in Section 4 of 
[4]: if $X$ is a $(b-1)$-connected spectrum, $n$ an integer $\geq b+2$, and if $r$ denotes the integer such that $2 r(p-1) \leq n-b-1<2(r+1)(p-1)$, then

$$
p^{r(r+1) / 2}\left(\operatorname{coker}_{n}\right)=0 .
$$

Remark 3.6. In Section 1 of [3], we gave universal upper bounds $R_{j+1}$ for the order of the $k$-invariants of connective spectra $E: R_{j+1} k^{j+1}(E)=0$ for all integers $j \geq 1$. Consequently, we may replace the conclusion of Theorem 3.2 by the following universal assertion (i.e., independent of $E$ ): for any connective spectrum $E$ and any $(b-1)$-connected spectrum $X$, the cokernel of the generalized E-Hurewicz homomorphism $h_{n}: E_{n}(X) \rightarrow H_{n}\left(X ; \pi_{0} E\right)$ satisfies

$$
\left(R_{2} R_{3} \cdots R_{n-b}\right)\left(\operatorname{coker} h_{n}\right)=0
$$

for all integers $n \geq b+2$.

The construction of the generalized $E$-Hurewicz homomorphism may even be done in a more general way in order to define homomorphisms

$$
h_{n, t}: E_{n}(X) \longrightarrow H_{n-t}\left(X ; \pi_{t} E\right)
$$

for a $(b-1)$-connected spectrum $X$, an integer $n \geq b$, and for all integers $t$ such that $0 \leq t \leq n-b: h_{n, t}$ is the homomorphism induced by the map of spectra $\lambda_{n, t}: E[n-b] \rightarrow$ $\Sigma^{t} H\left(\pi_{t} E\right)$ introduced in Theorem 1.5 of [4]. Observe that $h_{n, 0}=h_{n}$. We are also able to approximate the exponent of the cokernel of $h_{n, t}$, because Theorems 1.5 and 2.4 of [4] establishes the existence of a homomorphism $\Theta_{n, t}: H_{n-t}\left(X ; \pi_{t} E\right) \rightarrow E_{n}(X)$ with the property that the composition

$$
H_{n-t}\left(X ; \pi_{t} E\right) \stackrel{\Theta_{n, t}}{\longrightarrow} E_{n}(X) \stackrel{h_{n, t}}{\longrightarrow} H_{n-t}\left(X ; \pi_{t} E\right)
$$

is multiplication by the product $\rho_{t}(E) \rho_{t+1}(E) \cdots \rho_{n-b}(E)$ on $H_{n-t}\left(X ; \pi_{t} E\right)$.

Theorem 3.7. For any connective spectrum $E$ and any $(b-1)$-connected spectrum $X$, the cokernel of the generalized E-Hurewicz homomorphism $h_{n, t}: E_{n}(X) \rightarrow H_{n-t}\left(X ; \pi_{t} E\right)$ fulfills

$$
\rho_{t}(E) \rho_{t+1}(E) \cdots \rho_{n-b}(E)\left(\operatorname{coker} h_{n, t}\right)=0
$$

for all integers $n \geq b+1$ and for all integers $t$ such that $0 \leq t \leq n-b\left(\rho_{j}(E)\right.$ denotes the order of $k^{j+1}(E)$; notice that $\rho_{0}(E)=1$.) 


\section{The classical Hurewicz homomorphism}

The results of Section 2 (for $E=H(\mathbb{Z})$ ) and of Section 3 (for $E=S$ ) produce universal upper bounds (independent of $X$ ) for the exponent of both kernel and cokernel of the classical stable Hurewicz homomorphism

$$
h_{n}: \pi_{n} X \longrightarrow H_{n}(X ; \mathbb{Z})
$$

for any bounded below spectrum $X$ and for all integers $n$. Recall that $h_{n}$ is an isomorphism if $n=b$ and an epimorphism if $n=b+1$.

Theorem 4.1. For any $(b-1)$-connected spectrum $X$, the classical stable Hurewicz homomorphism $h_{n}: \pi_{n} X \rightarrow H_{n}(X ; \mathbb{Z})$ has the following properties:

(a) $\bar{\rho}_{n-b}\left(\operatorname{ker} h_{n}\right)=0$ for all integers $n \geq b+1$,

(b) $\bar{\rho}_{n-b-1}\left(\right.$ coker $\left.h_{n}\right)=0$ for all integers $n \geq b+2$,

where $\rho_{j}$ denotes the exponent of $\pi_{j} S$ and $\bar{\rho}_{m}$ the product $\rho_{1} \rho_{2} \cdots \rho_{m} \quad(j, m \geq 1)$.

Proof. Assertion (a) is given by Corollary 2.3 for $E=H(\mathbb{Z}$ ) and Assertion (b) is a consequence of Theorem 3.2 in the case $E=S$ and of Theorem 1.3.

Now, if $X$ is a $(b-1)$-connected $p$-local spectrum for a given prime number $p$, and if $n$ is an integer $\leq 2 p+b-4$, then $\bar{\rho}_{n-b}$ and $\bar{\rho}_{n-b-1}$ are not divisible by $p$ and we deduce the following Hurewicz isomorphism theorem.

Corollary 4.2. If $X$ is a $(b-1)$-connected $p$-local spectrum for a given prime number $p$, then the classical Hurewicz homomorphism $h_{n}: \pi_{n} X \rightarrow H_{n}(X ; \mathbb{Z})$ is an isomorphism for all integers $n$ such that $b \leq n \leq 2 p+b-4$.

Remark 4.3. If $X$ is a $(b-1)$-connected $p$-local spectrum for a given prime $p$ and $n$ an integer satisfying $b \leq n \leq 2 p+b-4$, then all $k$-invariants of $X[n]$ are trivial according to Theorem 1.5 of [3]. Therefore, it turns out that $X[n]$ is actually a wedge of Eilenberg-MacLane spectra:

$$
X[n] \simeq \bigvee_{t=b}^{n} \Sigma^{t} H\left(\pi_{t} X\right)
$$

If one applies Theorem 4.1 to the suspension spectrum of a CW-complex, one get the following improvement of Corollary 2.7 in the classical case (this has also been proved independently by J. Scherer, see [7]). 
Corollary 4.4. For any $(b-1)$-connected $C W$-complex $K$ with $b \geq 3$ and any integer $n$ such that $b+1 \leq n \leq 2(b-1)$, the classical Hurewicz homomorphism $h_{n}: \pi_{n} K \rightarrow$ $H_{n}(K ; \mathbb{Z})$ satisfies $\bar{\rho}_{n-b}\left(\operatorname{ker} h_{n}\right)=0$ and $\bar{\rho}_{n-b-1}\left(\operatorname{coker} h_{n}\right)=0$.

Let us conclude this section by looking at the classical unstable Hurewicz homomorphism $h_{*}$ for infinite loop spaces. Let $K$ be a $(b-1)$-connected infinite loop space with $b \geq 1$ and $X_{K}$ an associated $(b-1)$-connected $\Omega$-spectrum. For any integer $n \geq b$, consider the diagram

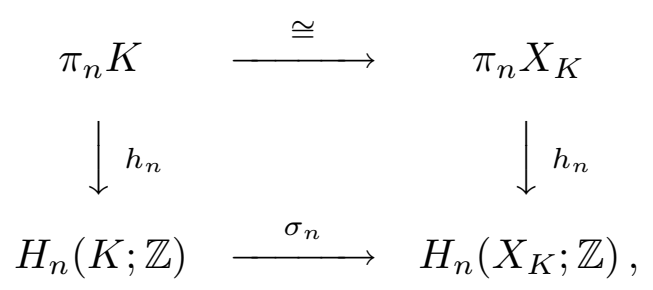

where $\sigma_{n}$ is the iterated homology suspension. In order to formulate the next result, define $\tilde{h}_{n}: \pi_{n} K \rightarrow H_{n}(K ; \mathbb{Z}) /\left(\operatorname{ker} \sigma_{n}\right)$ as the composition of the unstable Hurewicz homomorphism $h_{n}: \pi_{n} K \rightarrow H_{n}(K ; \mathbb{Z})$ with the quotient map $H_{n}(K ; \mathbb{Z}) \rightarrow H_{n}(K ; \mathbb{Z}) /\left(\operatorname{ker} \sigma_{n}\right)$, and write $\bar{\sigma}_{n}: \operatorname{coker}\left(h_{n}: \pi_{n} K \rightarrow H_{n}(K ; \mathbb{Z})\right) \rightarrow \operatorname{coker}\left(h_{n}: \pi_{n} X_{K} \rightarrow H_{n}\left(X_{K} ; \mathbb{Z}\right)\right)$ for the homomorphism induced by $\sigma_{n}$. The commutativity of the above diagram implies the following consequence of Theorem 4.1.

Corollary 4.5. If $K$ is a $(b-1)$-connected infinite loop space with $b \geq 1$, then the classical unstable Hurewicz homomorphism $h_{n}: \pi_{n} K \rightarrow H_{n}(K ; \mathbb{Z})$ satisfies:

(a) $\bar{\rho}_{n-b}\left(\right.$ ker $\left.h_{n}\right)=0$ for all integers $n \geq b+1$,

(b) $\bar{\rho}_{n-b-1}\left(\operatorname{coker} \tilde{h}_{n}\right)=0$ and $\bar{\rho}_{n-b-1}\left(\operatorname{coker} h_{n}\right) \subset \operatorname{ker} \bar{\sigma}_{n}$ for all integers $n \geq b+2$.

\section{The Hurewicz homomorphism in algebraic K-theory}

Let $R$ be any ring with identity, $G L(R)$ the infinite general linear group (considered as a discrete group) over $R, E(R)$ its subgroup generated by elementary matrices, and $B E(R)^{+}$the simply connected infinite loop space obtained by performing the plus construction on the classifying space of $E(R)$. Any simply connected $\Omega$-spectrum $X_{B E(R)^{+}}$ associated with $B E(R)^{+}$is of particular interest, because Corollary 4.2 shows that its homology detects the algebraic K-theory of $R$ at large primes. 
Corollary 5.1. For any ring $R$ and any integer $n \geq 2, K_{n}\left(R ; \mathbb{Z}_{(p)}\right) \cong H_{n}\left(X_{B E(R)^{+}} ; \mathbb{Z}_{(p)}\right)$ for all prime numbers $p \geq \frac{n}{2}+1$.

The relationship between algebraic K-theory and linear group homology is described by the Hurewicz homomorphism

$$
h_{n}: K_{n} R \cong \pi_{n} B E(R)^{+} \longrightarrow H_{n}\left(B E(R)^{+} ; \mathbb{Z}\right) \cong H_{n}(E(R) ; \mathbb{Z})
$$

for $n \geq 2$ (see [2] for other results on this homomorphism). Obviously, $h_{2}$ is an isomorphism and $h_{3}$ is surjective, and it is known that $2\left(\operatorname{ker} h_{3}\right)=0$ (see [8], p. 370, [6], Proposition 2.5, and [2], Corollary 1.8 and Remark 1.9). The next result follows directly from Corollary 4.5 and Corollary 5.1.

Corollary 5.2. For any ring $R$, the Hurewicz homomorphism $h_{n}: K_{n} R \rightarrow H_{n}(E(R) ; \mathbb{Z})$ has the following properties:

(a) $\bar{\rho}_{n-2}\left(\operatorname{ker} h_{n}\right)=0$ for all integers $n \geq 3$,

(b) for any integral homology class $x \in H_{n}(E(R) ; \mathbb{Z}), n \geq 4$, there exists an element $y$ in the image of $h_{n}$ and an element $z$ in the kernel of the iterated homology suspension $\sigma_{n}: H_{n}(E(R) ; \mathbb{Z}) \rightarrow H_{n}\left(X_{B E(R)^{+}} ; \mathbb{Z}\right)$ such that $\bar{\rho}_{n-3} x=y+z$,

(c) for any integer $n \geq 2, h_{n}: K_{n}\left(R ; \mathbb{Z}_{(p)}\right) \rightarrow H_{n}\left(E(R) ; \mathbb{Z}_{(p)}\right)$ is a split injection for all prime numbers $p \geq \frac{n}{2}+1$.

For small values of $n$, the odd primary part of the universal upper bound for the exponent of the kernel of $h_{n}$ given by Assertion (a) is better than the approximation obtained in [2]. However, note that the modifications mentioned in Remarks 2.5 and 3.6 may be used here again; in particular, we can replace Assertion (a) by: $\operatorname{gcd}\left(\bar{\rho}_{n-2}, R_{n-1}\right)\left(k e r h_{n}\right)=0$ for any ring $R$ and for all $n \geq 3$, where the integers $R_{n-1}$ are defined in [2] and [3].

Assertion (b) is a generalization of the description of the cokernel of $h_{n}$ we established for $n=4$ in Section 3 of [2]. Take for instance $R=\mathbb{Z}$ and $n=14$ : it is known that $K_{14} \mathbb{Z}$ is a finite abelian group and that $H_{14}(S L(\mathbb{Z}) ; \mathbb{Z}) \cong \mathbb{Z} \oplus$ (finite abelian group), because $K_{j} \mathbb{Z} \otimes \mathbb{Q}=0$ for $j \not \equiv 1 \bmod 4$ and $H^{*}(S L(\mathbb{Z}) ; \mathbb{Q}) \cong \Lambda\left(x_{5}, x_{9}, \ldots, x_{4 j+1}, \ldots\right)$ by $[5] ;$ then, a generator $x$ of the infinite cyclic summand of $H_{14}(S L(\mathbb{Z}) ; \mathbb{Z})$, which represents an element of coker $h_{14}$, has the property that $\sigma_{14}(x)$ is a torsion class. This situation happens more generally if $R$ is a number field or the ring of algebraic integers in a number field.

Observe that the integers $\bar{\rho}_{n-2}$ and $\bar{\rho}_{n-3}$ occuring in the statement of Corollary 5.2 are independent of the ring $R$. Similar results hold, of course, for the infinite general linear group $G L(R)$ and the infinite Steinberg group $S t(R)$ (by setting $b=1$, respectively $b=3$ in Corollary 4.5). 


\section{The Hurewicz homomorphism with finite coefficients}

Let us finally consider the E-Hurewicz homomorphism with coefficients in $\mathbb{Z} / p^{d}$ for a spectrum $X$ :

$$
h_{n}^{p^{d}}: \pi_{n}\left(X ; \mathbb{Z} / p^{d}\right) \cong M\left(\mathbb{Z} / p^{d}\right)_{n}(S \wedge X) \stackrel{(i \wedge \mathrm{id})_{*}}{\longrightarrow} M\left(\mathbb{Z} / p^{d}\right)_{n}(E \wedge X)=E\left(\mathbb{Z} / p^{d}\right)_{n}(X),
$$

where $p$ is a prime number, $d$ a positive integer, $E$ a connective ring spectrum with identity $i: S \rightarrow E, M\left(\mathbb{Z} / p^{d}\right)$ the $\bmod p^{d}$ Moore spectrum, and where $E\left(\mathbb{Z} / p^{d}\right)$ denotes $E \wedge M\left(\mathbb{Z} / p^{d}\right)$. In particular, for $E=H(\mathbb{Z})$, we get again the classical mod $p^{d}$ Hurewicz homomorphism

$$
h_{n}^{p^{d}}: \pi_{n}\left(X ; \mathbb{Z} / p^{d}\right) \longrightarrow H_{n}\left(X ; \mathbb{Z} / p^{d}\right),
$$

since $H(\mathbb{Z})\left(\mathbb{Z} / p^{d}\right)=H\left(\mathbb{Z} / p^{d}\right)$.

Definition 6.1. For $m \geq 1$, let $\bar{\rho}_{m, p^{d}}=\prod_{j=1}^{m} \rho_{j}\left(\mathbb{Z} / p^{d}\right)$, where $\rho_{j}\left(\mathbb{Z} / p^{d}\right)$ is the exponent of $\pi_{j} M\left(\mathbb{Z} / p^{d}\right)$.

The argument of the proof of Theorem 2.2 (in which $S$ should be replaced by $\left.M\left(\mathbb{Z} / p^{d}\right)\right)$ and Theorem $3.2\left(\right.$ for $E=M\left(\mathbb{Z} / p^{d}\right)$ ) together with Theorem 1.3 give the following result.

Theorem 6.2. Let $X$ be any $(b-1)$-connected spectrum, $p$ a prime number and $d$ a positive integer.

(a) For any connective ring spectrum $E$ with $\pi_{0} E$ torsion-free, if $x$ belongs to the kernel of the $\bmod p^{d} \quad E$-Hurewicz homomorphism $h_{n}^{p^{d}}: \pi_{n}\left(X ; \mathbb{Z} / p^{d}\right) \rightarrow E\left(\mathbb{Z} / p^{d}\right)_{n}(X)$, then

$$
\bar{\rho}_{n-b, p^{d}} x \otimes 1=0 \quad \text { in } \pi_{n}\left(X ; \mathbb{Z} / p^{d}\right) \otimes \pi_{0} E
$$

for all integers $n \geq b+1$.

(b) The cokernel of the classical mod $p^{d}$ Hurewicz homomorphism $h_{n}^{p^{d}}: \pi_{n}\left(X ; \mathbb{Z} / p^{d}\right) \rightarrow$ $H_{n}\left(X ; \mathbb{Z} / p^{d}\right)$ satisfies

$$
\bar{\rho}_{n-b-1, p^{d}}\left(\operatorname{coker} h_{n}^{p^{d}}\right)=0
$$

for all integers $n \geq b+2$. 


\section{References}

1. J.F. Adams: Stable homotopy and generalised homology (The University of Chicago Press, 1974).

2. D. Arlettaz: The Hurewicz homomorphism in algebraic K-theory, J. Pure Appl. Algebra $\mathbf{7 1}$ (1991), 1-12.

3. D. Arlettaz: The order of the differentials in the Atiyah-Hirzebruch spectral sequence, K-Theory 6 (1992), 347-361.

4. D. Arlettaz: Exponents for extraordinary homology groups, Comment. Math. Helv. 68 (1993), 653-672.

5. A. Borel: Cohomologie réelle stable de groupes S-arithmétiques classiques, C. R. Acad. Sci. Paris Sér. A 274 (1972), 1700-1702.

6. C.H. Sah: Homology of classical groups made discrete III, J. Pure Appl. Algebra 56 (1989), 269-312.

7. J. Scherer: Exponents for high-dimensional Gamma groups, preprint.

8. A.A. Suslin: Homology of $G L_{n}$, characteristic classes and Milnor K-theory, in Algebraic K-theory, number theory, geometry and analysis, Lecture Notes in Math. 1046 (Springer 1984), 357-375.

9. R.M. Switzer: Algebraic topology - homotopy and homology, Die Grundlehren der mathematischen Wissenschaften 212 (Springer 1975).

10. J.W. Vick: Poincaré duality and Postnikov factors, Rocky Mountain J. Math 3 (1973), 483-499.

Dominique Arlettaz

Institut de mathématiques

Université de Lausanne

CH-1015 Lausanne, Switzerland

e-mail: dominique.arlettaz@ima.unil.ch 